PROCEEDINGS OF THE

AMERICAN MATHEMATICAL SOCIETY

Volume 131, Number 9, Pages 2649-2655

S 0002-9939(03)06850-3

Article electronically published on January 8, 2003

\title{
DEGREE BOUNDS IN QUANTUM SCHUBERT CALCULUS
}

\author{
ALEXANDER YONG
}

(Communicated by John R. Stembridge)

\begin{abstract}
Fulton and Woodward have recently identified the smallest degree of $q$ that appears in the expansion of the product of two Schubert classes in the (small) quantum cohomology ring of a Grassmannian. We present a combinatorial proof of this result, and provide an alternative characterization of this smallest degree in terms of the rim hook formula for the quantum product.
\end{abstract}

\section{IntRoduCtion AND MAIN RESULtS}

Let $X=\operatorname{Gr}\left(l, \mathbb{C}^{n}\right)$ be the Grassmannian of $l$-dimensional subspaces in $\mathbb{C}^{n}$. The classical cohomology ring $\mathrm{H}^{*}(X, \mathbb{Z})$ has an additive basis of Schubert classes $\left\{\sigma_{\lambda}\right\}$, indexed by the Young diagrams $\lambda$ (identified with the corresponding partitions) contained in the $l \times k$ rectangle, where $k=n-l$ (we denote this by $\lambda \subseteq l \times k$ ). The product of two Schubert classes in $\mathrm{H}^{*}(X, \mathbb{Z})$ is given by

$$
\sigma_{\lambda} \cdot \sigma_{\mu}=\sum_{\nu \subseteq l \times k} c_{\lambda, \mu}^{\nu} \sigma_{\nu}
$$

where $c_{\lambda, \mu}^{\nu}$ is the Littlewood-Richardson coefficient (see, e.g., [6, 15]).

The (small) quantum cohomology ring $\mathrm{QH}^{*}(X)$ is a certain deformation of $\mathrm{H}^{*}(X, \mathbb{Z})$ that has been extensively studied in recent years; see, e.g., [1, 7, 14] and references therein.

The additive structure of $\mathrm{QH}^{*}(X)$ is essentially the same as that of $\mathrm{H}^{*}(X, \mathbb{Z})$ : the Schubert classes form a basis of $\mathrm{QH}^{*}(X)$ as a free module over $\mathbb{Z}[q]$, where $q$ is an indeterminate. The multiplicative structure of $\mathrm{QH}^{*}(X)$ is defined by

$$
\sigma_{\lambda} \star \sigma_{\mu}=\sum_{\nu \subseteq l \times k} \sum_{d \geq 0} q^{d}\left\langle\lambda, \mu, \nu^{\vee}\right\rangle_{d} \sigma_{\nu}
$$

where $\nu^{\vee}=\left(k-\nu_{l}, \ldots, k-\nu_{1}\right)$ is the complement of $\nu$ in the $l \times k$ rectangle, and $\left\langle\lambda, \mu, \nu^{\vee}\right\rangle_{d}$ is a three-point, genus-zero Gromov-Witten invariant of $X$. Note that we use " $\star$ " to distinguish the quantum multiplication from the product in the classical cohomology ring. Setting $q=0$ recovers (1) because $c_{\lambda, \mu}^{\nu}=\left\langle\lambda, \mu, \nu^{\vee}\right\rangle_{0}$.

Bertram, Ciocan-Fontanine and Fulton 2 have given a combinatorial rule to compute $\sigma_{\lambda} \star \sigma_{\mu}$ and thus the Gromov-Witten invariants $\left\langle\lambda, \mu, \nu^{\vee}\right\rangle_{d}$. To describe

Received by the editors December 14, 2001 and, in revised form, April 2, 2002.

1991 Mathematics Subject Classification. Primary 14M15; Secondary 05E05, 14N10.

Key words and phrases. Gromov-Witten invariants, quantum cohomology, Grassmannian, Schubert calculus. 

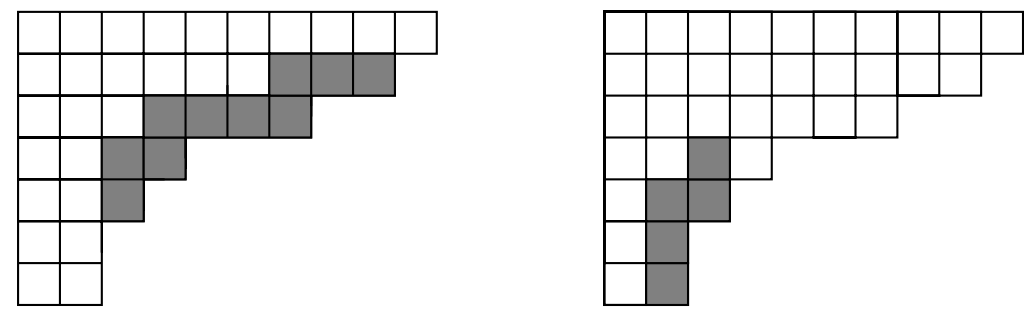

FIgURE 1. Legal and illegal rim hooks for $\lambda=(10,9,7,4,3,2,2)$

this rule, we need some terminology and notation. An n-rim hook of a Young diagram $\lambda$ is a connected subset of $n$ boxes of $\lambda$ that does not contain a $2 \times 2$ square. The width of an $n$-rim hook is the number of columns it occupies. An $n$-rim hook is legal if removing it from a Young diagram gives a valid Young diagram (see Figure $1)$. For a partition $\rho$, we define its $n$-core, denoted $\operatorname{core}_{n}(\rho)$, to be the partition corresponding to the Young diagram obtained by repeatedly removing legal $n$-rim hooks from $\rho$ until further removals are not possible. It is well known (see, e.g., [9]) that this procedure defines $\operatorname{core}_{n}(\rho)$ uniquely. Let $r_{n}(\rho)=\frac{|\rho|-\left|\operatorname{core}_{n}(\rho)\right|}{n}$ be the number of $n$-rim hooks removed in this process, and set $\epsilon(\rho)=(-1)^{\sum^{n}\left(k-\operatorname{width}\left(R_{i}\right)\right)}$, where $R_{1}, \ldots, R_{r_{n}(\rho)}$ are these $n$-rim hooks. With this notation, the rule obtained in [2] is as follows:

$$
\sigma_{\lambda} \star \sigma_{\mu}=\sum_{\nu \subseteq l \times k} \sum_{\substack{c_{\lambda, \mu}^{\rho} \neq 0 \\ \rho_{1} \leq k \\ \operatorname{cor}_{n}(\rho)=\nu}} q^{r_{n}(\rho)} \epsilon(\rho) c_{\lambda, \mu}^{\rho} \sigma_{\nu} .
$$

The Gromov-Witten invariants $\left\langle\lambda, \mu, \nu^{\vee}\right\rangle$ are known to be nonnegative; thus the coefficient of each term $q^{d} \sigma_{\nu}$ in the right-hand side of (3) is nonnegative, although no combinatorial proof of this fact is currently known.

For fixed $\lambda$ and $\mu$, let $\mathrm{d}_{\min }$ denote the smallest degree of $q$ such that $q^{\mathrm{d}_{\min }}$ appears in (2) with nonzero coefficient. The following theorem provides an affirmative answer to a question posed by A. Ram (private communication).

Theorem 1.1. Let $\lambda, \mu \subseteq l \times k$. Among all $\rho$ with $\rho_{1} \leq k$ and $c_{\lambda, \mu}^{\rho} \neq 0$, pick those with the largest $n$-core (equivalently, the smallest value of $r_{n}(\rho)$ ). Then their contributions to (3) do not cancel each other out. In other words, $\mathrm{d}_{\min }$ equals the smallest degree of $q$ that appears in the right-hand side of (31) (before cancellations).

Our proof of Theorem 1.1 will also prove a recent result of Fulton and Woodward (see Theorem 1.2 below). For partitions $\lambda, \mu \subseteq l \times k$, place $\lambda$ against the upper left corner of the rectangle. Then rotate $\mu 180$ degrees and place it in the lower right corner (see Figure 2). We will refer to rotate $(\mu)$ as the resulting subshape of $l \times k$. Let $\mathfrak{d}$ be the side length of the largest square that fits inside $\lambda \cap \operatorname{rotate}(\mu)$. The following theorem was conjectured by Fulton, and later proved by Fulton and Woodward [8], using moduli spaces 1$]$

Theorem $1.2([8]) . d_{\min }=\mathfrak{d}$.

\footnotetext{
${ }^{1}$ Actually, Fulton and Woodward proved a more general result that applies to any homogeneous space $X=G / P$, where $G$ is a simply connected complex semisimple Lie group and $P$ its parabolic subgroup.
} 


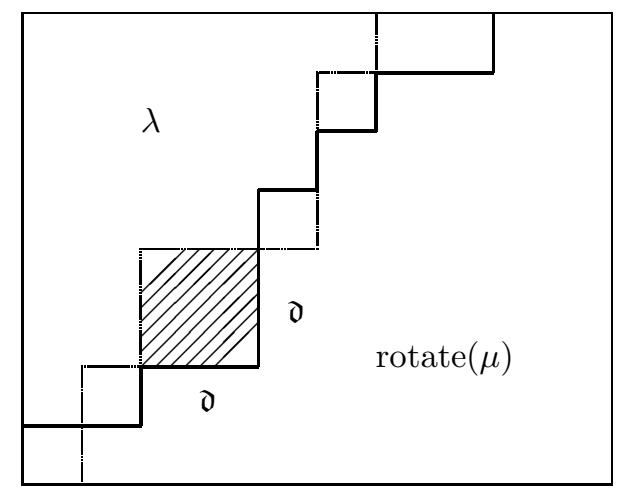

Figure 2. $\lambda, \operatorname{rotate}(\mu)$ and $\mathfrak{d} \times \mathfrak{d}$

An alternative proof of Theorem 1.2 was later given by A. Buch [3], using an elegant geometric argument combined with combinatorics. We will utilize the combinatorial part of Buch's proof below.

Let us now describe our proof of Theorems 1.1 and 1.2 The proof is entirely combinatorial once the nonnegativity of the Gromov-Witten invariants and the fact that $\mathrm{QH}^{*}(X)$ is an associative ring [10, 13] are granted. Our argument is based on the following result.

Theorem 1.3. Let $a \times A, b \times B$ and $c \times C$ be rectangular Young diagrams contained in $l \times k$. Then the following are equivalent:

(I) there exist Young diagrams $\lambda, \mu, \nu \subseteq l \times k$ containing $a \times A, b \times B$ and $c \times C$, respectively, such that $\sigma_{\lambda} \cdot \sigma_{\mu} \cdot \sigma_{\nu} \neq 0$;

(II) $\sigma_{a \times A} \cdot \sigma_{b \times B} \cdot \sigma_{c \times C} \neq 0$;

(III) all of the five conditions below hold:

(i) $a+b \leq l$ or $A+B \leq k$;

(ii) $a+c \leq l$ or $A+C \leq k$;

(iii) $b+c \leq l$ or $B+C \leq k$;

(iv) $a+b+c \leq l$ or $A+B+C \leq 2 k$;

(v) $a+b+c \leq 2 l$ or $A+B+C \leq k$.

A combinatorial proof of Theorem 1.3 is given in Section 2. S. Fomin (private communication) has observed that the necessity of conditions (i)-(v) can be derived from the Horn inequalities describing the "Klyachko cone" $\left\{(\lambda, \mu, \nu) \mid c_{\lambda, \mu}^{\nu} \neq 0\right\}$; specifically see [5, (11)].

Corollary 1.4. Let $\lambda, \mu, \rho$ be partitions such that $\lambda, \mu \subseteq l \times k$ and $\rho_{1} \leq k$. If $c_{\lambda, \mu}^{\rho} \neq 0$, then $(l+\mathfrak{d}) \times \mathfrak{d} \subseteq \rho$.

Proof. Suppose $c_{\lambda, \mu}^{\rho} \neq 0$ but $(l+\mathfrak{d}) \times \mathfrak{d} \nsubseteq \subseteq \rho$. Choose any positive integer $L \geq l$ such that $\rho \subseteq L \times k$. Then we have $\sigma_{\lambda} \cdot \sigma_{\mu} \cdot \sigma_{\rho^{\vee}} \neq 0$ in the classical cohomology ring of the corresponding Grassmannian. Notice that $(L-(l+\mathfrak{d})+1) \times(k-\mathfrak{d}+1) \subseteq \rho^{\vee}$. By the definition of $\mathfrak{d}$, there exist rectangles $a \times A \subseteq \lambda$ and $b \times B \subseteq \mu$ such that $a+b \geq l+\mathfrak{d}$ and $A+B \geq k+\mathfrak{d}$. Set $\nu=\rho^{\vee}, c=L-(l+\mathfrak{d})+1$ and $C=k-\mathfrak{d}+1$. Then it is easy to check that both corresponding inequalities (iv) are violated, a contradiction of Theorem 1.3 (see Figure 3). 


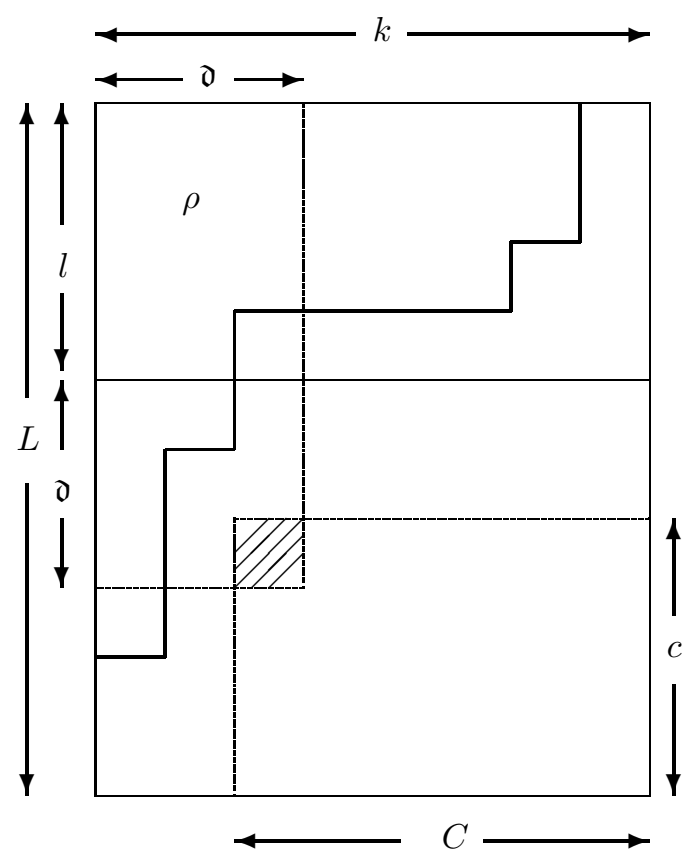

Figure 3. Proof of Corollary 1.4

Proof of Theorems 1.1 and 1.2. Let $\rho$ be a partition whose first part is at most $k$ and whose $n$-core is contained in $l \times k$. If $c_{\lambda, \mu}^{\rho} \neq 0$, then Corollary 1.4 gives $(l+\mathfrak{d}) \times \mathfrak{d} \subseteq \rho$, implying that at least $\mathfrak{d}$ rim hook removals are needed to obtain $\operatorname{core}_{n}(\rho)$. Hence the smallest degree of $q$ that occurs in (3), and therefore $\mathrm{d}_{\min }$, is at least $\mathfrak{d}$.

To complete the proofs, it remains to show that $\mathrm{d}_{\min }$ and thus the smallest degree of $q$ that appears in (3) is at most $\mathfrak{d}$. To this end, we borrow an argument of A. Buch [3, used in his own proof of Theorem 1.2. We reproduce his argument below:

Let $\tilde{\lambda}$ and $\bar{\lambda}$ be the partitions obtained by removing the leftmost $\mathfrak{d}$ columns and the top $\mathfrak{d}$ rows of $\lambda$, respectively. Also, let $\widetilde{\bar{\lambda}}$ be the partition obtained by removing both the leftmost $\mathfrak{d}$ columns and the top $\mathfrak{d}$ rows of $\lambda$. Set $\alpha=(k+\mathfrak{d}-$ $\left.\lambda_{\mathfrak{d}}, \ldots, k+\mathfrak{d}-\lambda_{1}\right)$ and let $\beta_{i}=\max \left(\mathfrak{d}-\lambda_{l+1-i}, 0\right)$ for $1 \leq i \leq l$. In other words, $\alpha$ is the complement of the bottom $\mathfrak{d}$ rows of $\operatorname{rotate}(\lambda)$ in $l \times(k+\mathfrak{d})$, and $\beta$ is the complement of $\operatorname{rotate}(\lambda)$ in the rightmost $\mathfrak{d}$ columns (see Figure 4 ).

It follows from the Littlewood-Richardson rule that the expansion of $\sigma_{\lambda} \star \sigma_{\beta}$ contains the class $\sigma_{\left(\mathfrak{o}^{l}\right)+\tilde{\lambda}}=\sigma_{\left(\mathfrak{d}^{l}\right)} \star \sigma_{\tilde{\lambda}}$. It also follows that $\sigma_{\tilde{\lambda}} \star \sigma_{\alpha}$ contains $\sigma_{\left(k^{\mathfrak{d}}\right), \tilde{\lambda}}=$ $\sigma_{\left(k^{\mathfrak{d}}\right)} \star \sigma_{\tilde{\lambda}}$. It is not hard to check directly from (3) that $\sigma_{\left(\mathfrak{d}^{l}\right)} \star \sigma_{\left(k^{\mathfrak{d}}\right)}=q^{\mathfrak{d}}$. By the nonnegativity of the Gromov-Witten invariants and the associativity of $\mathrm{QH}^{*}(X)$, $\sigma_{\lambda} \star \sigma_{\beta} \star \sigma_{\alpha}$ contains the product $\sigma_{\left(\mathfrak{d}^{l}\right)} \star \sigma_{\left(k^{\mathfrak{d}}\right)} \star \sigma_{\widetilde{\lambda}}=q^{\mathfrak{d}} \sigma_{\tilde{\bar{\lambda}}}$. Note that $\widetilde{\bar{\lambda}} \cap \operatorname{rotate}(\mu)=\emptyset$, which is well known to be equivalent to $\sigma_{\tilde{\lambda}} \cdot \sigma_{\mu} \neq 0$. Thus we conclude that $\sigma_{\lambda} \star \sigma_{\mu} \star \sigma_{\alpha} \star \sigma_{\beta}$ contains $q^{\mathfrak{d}}$ times some Schubert class. Therefore, the product $\sigma_{\lambda} \star \sigma_{\mu}$ must have a term involving a degree of $q$ less than or equal to $\mathfrak{d}$, and we are done. 


$$
\begin{aligned}
& A \cup B \cup C \cup D=\operatorname{rotate}(\lambda) \\
& A \cup B=\operatorname{rotate}(\bar{\lambda}) \\
& A \cup C=\operatorname{rotate}(\widetilde{\lambda}) \\
& A=\operatorname{rotate}(\widetilde{\bar{\lambda}}) \\
& D=\operatorname{rotate}(\mathfrak{d} \times \mathfrak{d})
\end{aligned}
$$

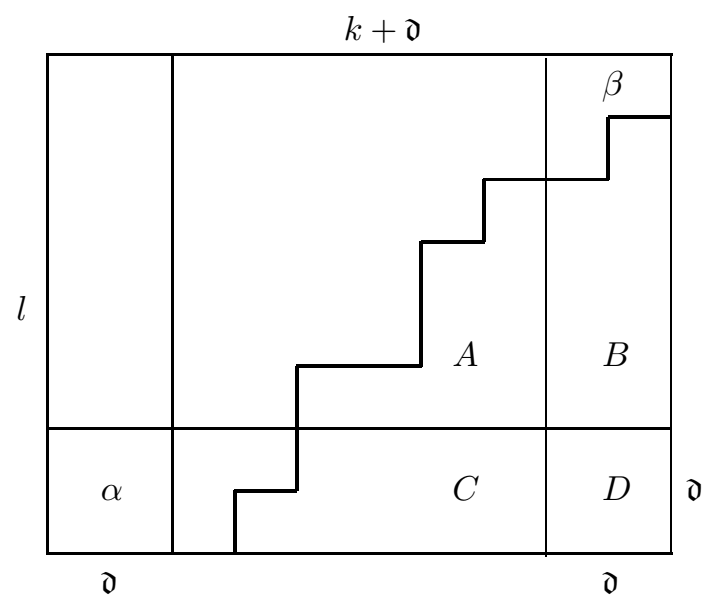

Figure 4. Proof of Theorems 1.1 and 1.2

\section{Proof of Theorem 1.3}

For $\alpha, \beta \subseteq l \times k$, define $\kappa(\alpha, \beta)$ to be the intersection of all Young diagrams $\gamma \subseteq l \times k$ such that $c_{\alpha, \beta}^{\gamma} \neq 0$. In the event the set of such $\gamma$ is empty, we set $\kappa(\alpha, \beta)=l \times k$ by convention. We will make use of the following observation, which follows immediately from this definition:

Lemma 2.1. Let $\alpha, \beta, m \times M \subseteq l \times k$ and $m \times M$ be nonempty. Then $\sigma_{\alpha} \cdot \sigma_{\beta} \cdot \sigma_{m \times M} \neq$ 0 if and only if $\kappa(\alpha, \beta) \cap \operatorname{rotate}(m \times M)=\emptyset$.

Our proof of Theorem 1.3 also uses the following two lemmas

Lemma 2.2. Let $\alpha, \beta, \lambda, \mu \subseteq l \times k$ be such that $\alpha \subseteq \lambda$ and $\beta \subseteq \mu$. Then $\kappa(\alpha, \beta) \subseteq$ $\kappa(\lambda, \mu)$.

Proof. It suffices to prove the case when $\lambda=\alpha$ and $\mu \backslash \beta$ is a single box. Since every term of $\left(\sigma_{\alpha} \cdot \sigma_{\beta}\right) \cdot \sigma_{1}$ is indexed by a partition containing $\kappa(\alpha, \beta)$, the same is true for $\sigma_{\alpha} \cdot\left(\sigma_{\beta} \cdot \sigma_{1}\right)=\sigma_{\lambda} \cdot\left(\sigma_{\mu}+\right.$ nonnegative terms $)$. The claim follows.

The next lemma is proved by a straightforward application of the LittlewoodRichardson Rule. Details are left to the reader.

Lemma 2.3. Let $m \times M, n \times N \subseteq l \times k$. If $\sigma_{m \times M} \cdot \sigma_{n \times N}=0$, then $\kappa(m \times M, n \times N)=$ $l \times k$. Otherwise,

$$
\begin{aligned}
\kappa(m \times M, n \times N)= & (m \times M) \cup(n \times N) \\
& \cup((m+n) \times(M+N-k)) \\
& \cup((m+n-l) \times(M+N)),
\end{aligned}
$$

where each of the last two rectangles in the right-hand side is understood to be empty if one of its dimensions is negative, or if it does not fit inside $l \times k$.

\footnotetext{
${ }^{2}$ Although we will not need it here, Lemma 2.2 generalizes to a statement about the cohomology of any homogeneous space $G / P$. The proof is analogous to the one given above.
} 
Proof of Theorem 1.3. We may assume that $c \times C$ is nonempty. Suppose (I) holds. Then there exists $\rho \subseteq l \times k$ with $c_{\lambda, \mu}^{\rho} \neq 0$ and $\rho \cap \operatorname{rotate}(\nu)=\emptyset$. Hence $\kappa(\lambda, \mu) \cap$ $\operatorname{rotate}(\nu)=\emptyset$ which by Lemma 2.2 implies

$$
\kappa(a \times A, b \times B) \cap \operatorname{rotate}(c \times C)=\emptyset .
$$

By Lemma 2.1. (44) is equivalent to (II), which itself trivially implies (I). Lastly, it follows from Lemma [2.3] that (4) is equivalent to (III).

\section{AN UPPER BOUND, OPEN PROBLEMS AND CONJECTURES}

It is an open problem to determine $\mathrm{d}_{\max }$, the largest degree of $q$ such that $q^{\mathrm{d}_{\max }}$ appears in (2) with nonzero coefficient. In particular, $\mathrm{d}_{\max }$ is not equal to the largest degree of $q$ appearing in (3). Next, we present a simple upper bound for $\mathrm{d}_{\max }$. For a Young diagram $\alpha$, let $\operatorname{diag}(\alpha)$ be the size of the largest square contained in $\alpha$.

Proposition 3.1. $\mathrm{d}_{\max } \leq \min (\operatorname{diag}(\lambda), \operatorname{diag}(\mu))$.

We note that for a large class of pairs $(\lambda, \mu)$, this inequality is sharper than the obvious upper bound $\mathrm{d}_{\max } \leq \frac{|\lambda|+|\mu|}{n}$.

Proof of Proposition 3.1. Let $\lambda$ be a Young diagram and $\lambda=\left(\alpha_{1}, \ldots, \alpha_{t} \mid \beta_{1}, \ldots, \beta_{t}\right)$ its Frobenius notation; the following is a well-known identity for Schur functions (see, e.g., [11]):

$$
s_{\lambda}=\operatorname{det}\left(s_{\left(\alpha_{i} \mid \beta_{j}\right)}\right)_{1 \leq i, j \leq t} .
$$

From (5) and our description of $\mathrm{QH}^{*}(X)$ it follows that

$$
\sigma_{\lambda}=\operatorname{det}\left(\sigma_{\left(\alpha_{i} \mid \beta_{j}\right)}\right)_{1 \leq i, j \leq t} .
$$

It is easy to check from the Littlewood-Richardson rule that for any $(\alpha \mid \beta), \lambda \subseteq l \times k$, the largest degree of $q$ that appears in $\sigma_{(\alpha \mid \beta)} \star \sigma_{\lambda}$ is 1 . By (6) we have

$$
\sigma_{\lambda} \star \sigma_{\mu}=\operatorname{det}\left(\sigma_{\left(\alpha_{i} \mid \beta_{j}\right)}\right)_{1 \leq i, j \leq t} \star \sigma_{\mu}=\sum_{\tau \in \mathcal{S}_{n}} \operatorname{sign}(\tau)\left(\prod_{i=1}^{t} \sigma_{\left(\alpha_{i} \mid \beta_{\tau(i)}\right)}\right) \star \sigma_{\mu} .
$$

Thus $\mathrm{d}_{\max } \leq \operatorname{diag}(\lambda)$. Switching the roles of $\lambda$ and $\mu$ gives $\mathrm{d}_{\max } \leq \operatorname{diag}(\mu)$.

It is an interesting problem to determine precisely which degrees of $q$ appear in the product $\sigma_{\lambda} \star \sigma_{\mu}$. Based on extensive computational evidence, we conjecture:

Conjecture 3.2. The product $\sigma_{\lambda} \star \sigma_{\mu}$ involves $q^{d}$ for any $d \in\left[\mathrm{d}_{\min }, \mathrm{d}_{\max }\right] 3^{3}$

More seems to be true.

Conjecture 3.3. Let $d \geq 1$ be an integer. If $\langle\lambda, \mu, \nu\rangle_{d} \neq 0$, then either there exists $\alpha \subseteq \nu$ such that $\langle\lambda, \mu, \alpha\rangle_{d-1} \neq 0$, or else $\langle\lambda, \mu, \alpha\rangle_{j}=0$ for all $\alpha \subseteq l \times k$ and all $0 \leq j \leq d-1$.

\footnotetext{
${ }^{3}$ Note added in proof. This conjecture has been proved by A. Postnikov [12].
} 


\section{ACKNOWLEDGMENTS}

This work was partially carried out while the author was visiting the Fields Institute in Toronto, and later the Isaac Newton Institute in Cambridge. We thank both Institutes for their hospitality. We are most deeply indebted to Sergey Fomin, whose guidance greatly improved the content and form of this paper. We benefited from conversations with Anders Buch, Bill Fulton, Arun Ram and Chris Woodward. Most of our computational investigations were done using Anders Buch's package [4].

\section{REFERENCES}

[1] A. Bertram, Quantum Schubert Calculus, Adv. Math. 128 (1997) 289-305. MR 98j:14067

[2] A. Bertram, I. Ciocan-Fontanine and W. Fulton, Quantum Multiplication of Schur polynomials, Journal of Algebra 219 (1999) 728-746. MR 2000k:14042

[3] A. Buch, Quantum cohomology of Grassmannians, e-print math.AG/0106268.

[4] A. Buch, Littlewood-Richardson Calculator, software available at http://www-math. mit.edu/ abuch.

[5] W. Fulton, Eigenvalues, invariant factors, highest weights, and Schubert calculus, Bull. Amer. Math. Soc. (N.S.) 37 (2000), no. 3, 209-249. MR 2001g:15023

[6] W. Fulton, Young tableaux, Cambridge University Press, 1997. MR 99f:05119

[7] W. Fulton and R. Pandharipande, Notes on stable maps and quantum cohomology, Algebraic geometry—Santa Cruz 1995, Amer. Math. Soc., Providence, RI, 1997, 45-96. MR 98m:14025

[8] W. Fulton and C. Woodward, preprint, 2001.

[9] G. James and A. Kerber, The representation theory of the symmetric group, Encyclopedia of Mathematics and its Applications, 16. Addison-Wesley Co. Reading, Mass., 1981.

[10] M. Kontsevich and Y. Manin, Gromov-Witten classes, quantum cohomology, and enumerative geometry, Mirror Symmetry II, Amer. Math. Soc., Providence, RI, 1997, 607-653.

[11] I. Macdonald, Symmetric functions and Hall polynomials, Second edition, Oxford University Press, 1995. MR 96h:05207

[12] A. Postnikov, Affine approach to quantum Schubert calculus, e-print math. CO/0205165.

[13] Y. Ruan and G. Tian, A mathematical theory of quantum cohomology, Math. Res. Lett. 1 (1994) 269-278.

[14] F. Sottile, Rational Curves on Grassmannians: systems theory, reality, and transverality, to appear in Contemporary Mathematics, 2001.

[15] R. Stanley, Enumerative Combinatorics, Volume 2, Cambridge University Press, 1999. MR 2000k:05026

Department of Mathematics, University of Michigan, Ann Arbor, Michigan 48109

E-mail address: ayong@umich.edu 